\title{
Screening a Case Base for Stroke Disease Detection
}

\author{
José Neves ${ }^{1(凶)}$, Nuno Gonçalves ${ }^{2}$, Ruben Oliveira ${ }^{2}$, Sabino Gomes ${ }^{2}$, \\ João Neves ${ }^{3}$, Joaquim Macedo ${ }^{1}$, António Abelha ${ }^{1}$, César Analide ${ }^{1}$, \\ José Machado ${ }^{1}$, Manuel Filipe Santos ${ }^{1}$, and Henrique Vicente ${ }^{1,4}$ \\ ${ }^{1}$ Centro Algorithmi, Universidade do Minho, Braga, Portugal \\ \{jneves, macedo, abelha, analide, jmac\}@di.uminho.pt, \\ mfs@dsi.uminho.pt \\ 2 Departamento de Informática, Universidade do Minho, Braga, Portugal \\ \{pg24168,pg24166\}@alunos.uminho.pt, \\ sabinogomes.antonio@gmail.com \\ 3 Drs. Nicolas and Asp, Dubai, United Arab Emirates \\ joaocpneves@gmail.com \\ ${ }^{4}$ Departamento de Química, Escola de Ciências e Tecnologia, \\ Universidade de Évora, Évora, Portugal \\ hvicente@uevora.pt
}

\begin{abstract}
Stroke stands for one of the most frequent causes of death, without distinguishing age or genders. Despite representing an expressive mortality figure, the disease also causes long-term disabilities with a huge recovery time, which goes in parallel with costs. However, stroke and health diseases may also be prevented considering illness evidence. Therefore, the present work will start with the development of a decision support system to assess stroke risk, centered on a formal framework based on Logic Programming for knowledge representation and reasoning, complemented with a Case Based Reasoning (CBR) approach to computing. Indeed, and in order to target practically the CBR cycle, a normalization and an optimization phases were introduced, and clustering methods were used, then reducing the search space and enhancing the cases retrieval one. On the other hand, and aiming at an improvement of the CBR theoretical basis, the predicates`attributes were normalized to the interval $0 \ldots 1$, and the extensions of the predicates that match the universe of discourse were rewritten, and set not only in terms of an evaluation of its Quality-of-Information (QoI), but also in terms of an assessment of a Degree-of-Confidence (DoC), a measure of one's confidence that they fit into a given interval, taking into account their domains, i.e., each predicate attribute will be given in terms of a pair (QoI, DoC), a simple and elegant way to represent data or knowledge of the type incomplete, self-contradictory, or even unknown.
\end{abstract}

Keywords: Stroke Disease $\cdot$ Logic Programming $\cdot$ Knowledge Representation and Reasoning $\cdot$ Case Based Reasoning · Similarity Analysis 